\title{
SATELLITE IMAGERY ASSISTED ROAD-BASED VISUAL NAVIGATION SYSTEM
}

\author{
A. Volkova ${ }^{\text {a }}$, P.W. Gibbens ${ }^{\mathrm{b}}$, \\ ${ }^{a}$ Student Member, IEEE, PhD candidate, School of Aerospace, Mechanical and Mechatronic Engineering, \\ The University of Sydney, Australia - avo19544@uni.sydney.edu.au \\ ${ }^{\mathrm{b}}$ Associate Professor, School of Aerospace, Mechanical and Mechatronic Engineering, \\ The University of Sydney, Australia - peter.gibbens@ sydney.edu.au
}

KEY WORDS: Unmanned aerial vehicle (UAV), Navigation, Vision, Accurate road centreline extraction, Feature-based visual navigation, Splines

\begin{abstract}
:
There is a growing demand for unmanned aerial systems as autonomous surveillance, exploration and remote sensing solutions. Among the key concerns for robust operation of these systems is the need to reliably navigate the environment without reliance on global navigation satellite system (GNSS). This is of particular concern in Defence circles, but is also a major safety issue for commercial operations. In these circumstances, the aircraft needs to navigate relying only on information from on-board passive sensors such as digital cameras. An autonomous feature-based visual system presented in this work offers a novel integral approach to the modelling and registration of visual features that responds to the specific needs of the navigation system. It detects visual features from Google Earth ${ }^{\dagger}$ to build a feature database. The same algorithm then detects features in an on-board cameras video stream. On one level this serves to localise the vehicle relative to the environment using Simultaneous Localisation and Mapping (SLAM). On a second level it correlates them with the database to localise the vehicle with respect to the inertial frame.

The performance of the presented visual navigation system was compared using the satellite imagery from different years. Based on comparison results, an analysis of the effects of seasonal, structural and qualitative changes of the imagery source on the performance of the navigation algorithm is presented.
\end{abstract}

\section{INTRODUCTION}

Unmanned aerial vehicles (UAVs) are currently seen as an optimal solution for intelligence, surveillance and reconnaissance (ISR) missions of the next generation. Compared to humanoperated flights, UAVs offer more flexibility and allow for higher risk and are generally less expensive. Employed for various tasks from urban planning and management to exploration and mapping, most unmanned aerial systems have become highly dependent on the accuracy of their navigation system. Moreover, on surveillance and investigation missions such vehicles are subjected to the risk of losing its primary source of navigation information, GNSS due to jamming, interference, unreliability, or partial or complete failure. Commercial operations in environments such as so-called urban canyons where GNSS may be unreliable or inaccurate due to multi-path or occlusion, robust operation of the navigation system becomes a serious safety issue and other, preferably passive, sensors become necessary for robustness.

To allow tolerance to GNSS faults, a UAV needs to be given a capability to maintain its course and continuously localise relying on a backup passive navigation information source like a visual navigation system (VNS). Since the acceptable precision of on-board inertial-based navigation system is limited to relatively short periods of time due to the integration of sensor measurements containing errors, a regular update, usually provided by GNSS, is required. With the satellite information being potentially unavailable in an uncharacterised environment, a position update can be generated by a VNS coupled with simultaneous localisation and mapping (SLAM). Visual features detected in the image, registered in database can provide an instantaneous position update that limits the localisation uncertainty of the inertial solution to a minimum.

\footnotetext{
* Corresponding author

${ }^{\dagger}$ The algorithm is independent of the source of satellite imagery imagery and another provider can be used
}

Aerial imagery contains all the necessary information about the position and motion of the aircraft. Recently, the research community has been focused on developing methods to retrieve this information from imagery by means of feature-based extraction. While methods developed for Micro Aerial Vehicles (MAVs) mostly use Scale-Invariant Feature Transform (SIFT) or SpeededUp Robust Feature (SURF) [1-3] feature matching algorithms, the algorithms developed primarily for geographic information system (GIS) update show a semantic, or meaningful, approach to feature extraction. Although it has been recently shown that real-time motion tracking based on small image patches can be very precise [4], the use of such features for SLAM and data association on level flight over repeatable terrain has not been investigated.

As the research in the field of remote sensing shows, high-level visually identifiable features, such as roads, rooves, water bodies etc., can be reliably extracted and used to update the map information or extract road networks from high-resolution satellite imagery. Despite the abundance of GIS update methods offered, only a few approaches can be regarded as autonomous and suitable for real-time application [5]. Within the described framework, this article presents a visual navigation system that, due to efficient feature modelling, achieves a near real-time performance. The basic concept behind this is the detection, extraction, localisation and matching of high-level features present in the aerial imagery (road network and its components, areas of greenery, water bodies etc.) by modelling them with minimal geometric characterisations used for storage and association. The semantic features listed above are discussed in the paper as separate feature-tracking threads, which can run in parallel, contributing to the interpretation of the scene. A position update would be produced based on the information from the most reliable or the currently active thread. The focus of the current work has been on development of robust feature extraction and modelling that takes into account the a-priori knowledge about the road networks that 
suits the requirements of the navigation system.

\section{RELATED WORK}

The most complete review on the topic of road extraction was presented in Mena [5] and an elaborate comparison of various road extraction methods was conducted by Mayer [6]. A recent survey of the road extraction algorithms satisfying the requirements of visual aerial navigation systems can be found in Volkova [7]. Below, a brief overview of the feature extraction approaches is provided, focusing on algorithms designed for navigational purposes.

Research on the visual-aided navigation has been ongoing for more than two decades [8]. Typical road extraction approaches for the update of map and GIS information are designed as pipelines consisting of image segmentation, extraction and connection of road candidates and final network refinement. SVM classifier [9-12], tensor voting feature detector [13-16] and non-maximum suppression for road centreline extraction [16-23] have been popular road network update techniques. Although, these techniques are superior in quality to direct intensity-based classification and mathematical morphology, they are much more computationally demanding.

Recent corner-based and patch-based real-time motion estimation approaches $[4,24,25]$ for MAVs achieved high robustness in scenes with high-frequency self-similar texture. A UAV Navigation System presented in [27] combined point-based visual odometry with edge-based image registration. Since low-level features used in odometry-based algorithms are often not unique and can only be used in conjunction with depth information, they are useful in the short-term especially micro and mid-scale platforms but cannot be the sole basis of a SLAM-based visual navigation systems on a larger scale.

Visual navigation using higher level features (houses, roads, etc.) has been the focus of far fewer research works, partially due to a variety of features representing any one class. Such GIS features as lines (roads), points (road intersections) and regions (forests, lakes, buildings) were suggested for use in navigational system $[22,28]$ with special attention given to intersections $[29,30]$. The rest of this section provides an overview of the approaches that, in the authors' opinion, are most relevant to the current research. Vision systems focused on landmark detection in [19] utilised a combination of SURF-based image registration and road and building detection using Haar classifiers. Haar training involves creation of a large dataset of the buildings regions and road intersections. Although the comparison presented in the above work showed that the Haar classifier outperformed line-based intersection detectors and edge-based building detectors under various illumination conditions, its inability to deal with rotation increased the complexity of the system. The GIS-based system presented in [22] registered meaningful object-level features such as road centrelines, intersections and villages in real-time aerial imagery with the data of geographic information system (GIS). The road was extracted using Local Weighted Features (LWF), an approach to estimate the background value of a pixel based on local neighbourhood pixels. Subsequently, road end points, branch points and cross points were generated from extracted road networks and were matched with a GIS database.

Three-stage landmark detection navigation proposed in [31] extracted a few (3-10) significant objects per image, such as rooves of buildings, parking lots etc., based on pixel intensity level and the number of the pixels in the object. For each of the extracted objects the feature signature was calculated, defined as a sum of the pixel intensity values in radial directions for a sub-image enclosing the feature. The centroids of the extracted objects were simultaneously used to form a waypoint polygon. The angles between centroids and ratios of polygon sides to its perimeter were then used as scale and rotation-invariant features describing a waypoint in the database.

The autonomous map-aided visual navigation system proposed in this paper combines intensity and frequency-based segmentation, high-level feature extraction and feature pattern matching to achieve reliable feature registration and generate the position and orientation innovations restricting the inertial drift of the onboard navigation system.

\section{AUTOMATIC ROAD FEATURE EXTRACTION AND MATCHING (ARFEM) ALGORITHM}

This paper has the goal to provide on-board inertial navigation system with a localisation update calculated from the match between localised visual features registered in an image and a precomputed database. The proposed multi-pronged architecture of the feature-extraction algorithm is shown on Fig.1. Although the overall structure of the system involves detection of features of greenery and water classes, the specific focus of this paper is on the road-detection component. To generate localisation update, an automatic Road Feature Extraction and Matching algorithm has been developed. The algorithm analyses each image frame to detect the features belonging to one of several classes. It then refines, models, and localises the features and finally matches it to a database built using the same algorithm from Google Earth imagery. In the following section each of these steps of the algorithm is detailed.

\subsection{Image classification}

The first stage of the feature detection algorithm is intensitybased image segmentation. A maximum likelihood classifier trained on 3-5 images for each class was used to detect road, greenery and water regions in the image based on pixel colour, colour variance and frequency response (for the latter two classes). The resulting class objects were taken through the pipeline shown in Fig.1 to minimise the misclassification and improve the robustness of feature generation. This process is described in detail as the following.

The aerial image was classified into road, greenery, water, and background regions using the training data for each class. While road class training was based on intensity of the pixels only (Fig.2), the greenery class description also contains Gabor frequency response of the provided training region that allows discriminating it from water, which is similar in intensity. At the current stage of algorithm development objects of greenery and water classes

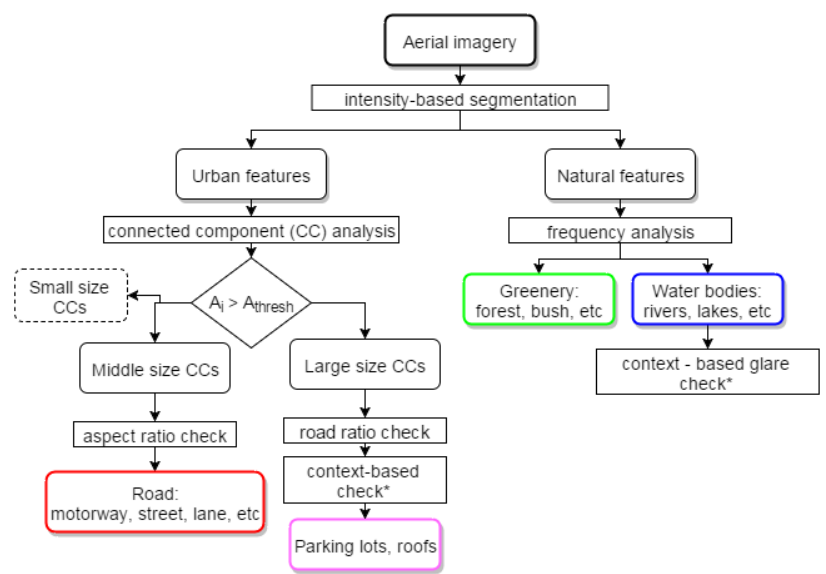

Figure 1: Image classification for visual feature extraction 
are used for detection of the environment in which the system is operating to adapt the detection techniques accordingly. Future realisation of the system will include processing threads for the corresponding classes and incorporation of localisation information derived from them.

\subsection{Road class filtering}

Since reliance on training only in image classification can result in misclassification, further filtering of the classes based on apriori knowledge about the nature of features in each class is performed. The most probable misclassification is between regions of confined water and greenery, and inclusion of road-like objects (parking lots, roofs) into the road components. To extract the road candidates from the urban features category of classes, connected component (CC) analysis [32] was used. The components were analysed with respect to size and compared with a threshold $A_{\text {thresh }}$. Components smaller than the threshold were discarded. Middle sized features with high bounding ellipse aspect ratio [16] were selected. Aspect ratio (shown on Fig.3) is calculated as follows.

$$
A R_{i}=\frac{a_{i}}{b_{i}}>>t_{A R},
$$

where $t_{A R}$ is an aspect ratio threshold. Aspect ratio [16] eliminates the misclassification in the road class due to inclusion of rooves and other non-road objects. In cases, where a bounding ellipse is drawn around a curved road segment (Fig.3, centre), the ellipse semi-minor axis is no longer a good approximation to the width of the road. To prevent such components from being discarded, a road ratio check is applied. The road ratio $R R_{i}$ is calculated by estimating the ratio of the road pixels to the total number of pixels within the bounding ellipse:

$$
R R_{i}=\frac{\text { road pixels }}{\text { bounding ellipse area }}>>t_{R R}
$$
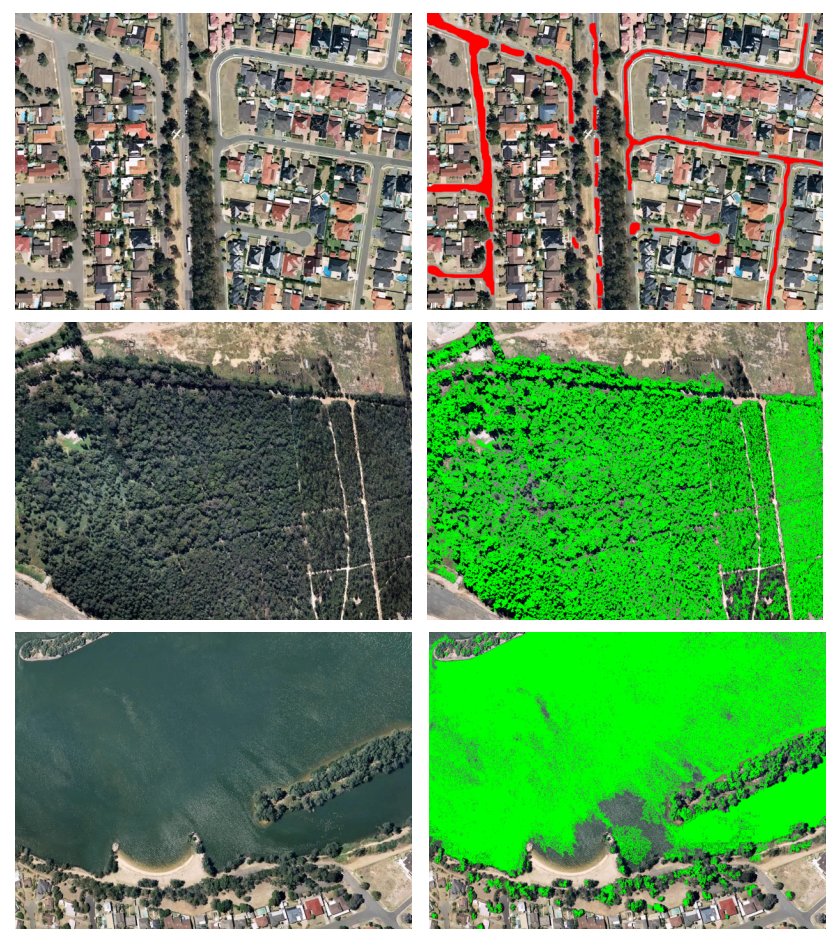

Figure 2: Training images and ground truth shown for road, greenery and water classes
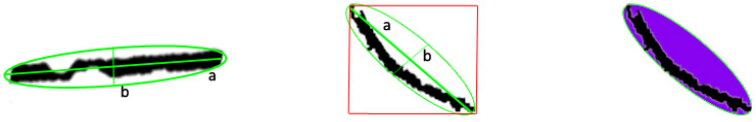

Figure 3: Comparison of bounding ellipses with bounding box method for straight (left) and curved road components (centre); the area of bounding ellipse in lilac compared to the area of the road component in black(right)

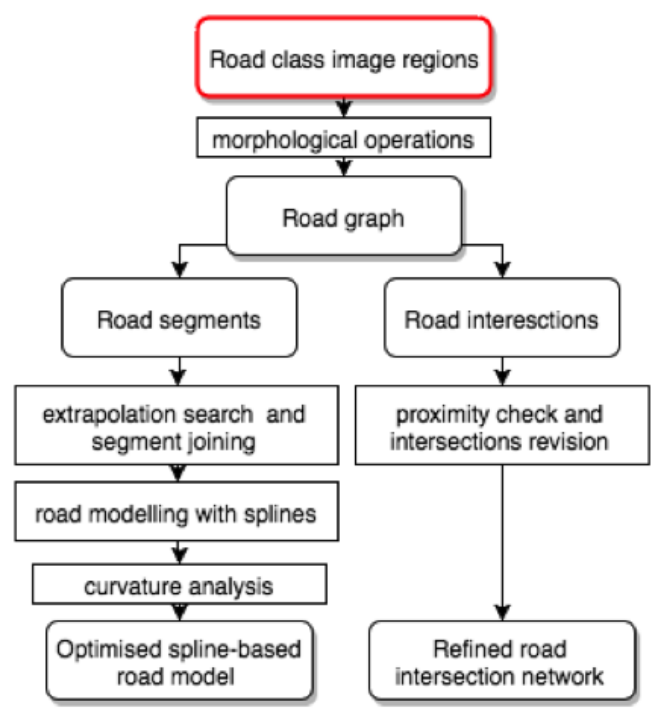

Figure 4: Flowchart of road network generation from road components detected in the image

For parking lots the road ratio would be considerably larger than the empirically defined threshold $t_{R R}$, road segments in turn would have a relatively low RR. The generated road component was processed with trivial morphological operations to improve the robustness of the centreline generation.

Natural features that include water bodies, forests, bush etc. are analysed using frequency analysis. Generally, water areas give higher frequency response, which allows for discrimination between the two. Glare present on the water remains one of the major segmentation problems for intensity-based approaches. Although further frequency analysis can be effective in glare detection it is a more computationally demanding operation compared to contextual solution.

We propose to distinguish between glare and other features similar in intensity based on the surrounding or neighbouring connected components and assign the glare region to the same class. For example, if a glare region is found in the image within the water region but comes up as a road-similar component based on its intensity, it can be filtered from the road class and inserted into the water class (processes marked with * in Fig.1). This glare processing routing works with the underlying assumption that there are no built-up areas or islands in the confined water regions.

\subsection{Road centreline extraction and extrapolation}

After road class image regions have been detected, the 2nd stage of the algorithm, outlined in Fig.4, converts them into a road network with defined centrelines and intersections. The road centreline candidates are derived from the filtered segmented road component by morphological thinning. Skeletonisation of the road component can be alternatively performed by non-maximum suppression [16, 17] and/or tensor voting algorithms [13-15]. Applying mathematical morphology [32] to the road skeleton, a road 
graph that describes the location and the length of the road segments together with road intersections is obtained. Further analysis and post-processing of road segments and intersections leads to a reliable road network description for further data association.

3.3.1 Extrapolation-based segment joining Some of the road branches in the road graph appear to be incomplete because of the occlusion or the rapid intensity change of the road surface in the image. To address these shortcomings, the following road branch search and connection method is proposed. Splines were fitted to the branch points and then extrapolated in the direction outward from the tip of the branch (Fig.5). The search areas (marked in red) were then checked for the presence of the tips of other branches. In case a tip of another branch is found within the search region, the algorithm suggests joining the branches. If the search initiated from the opposite branch finds the first branch, the branches will be joined.

3.3.2 Road centreline modelling with splines Road branches obtained in the previous stage are heavily influenced by road occlusions. For instance, in the presence of the trees along the road side, the road component will decrease in width and therefore its centreline will be shifted to the side opposite to occlusions. To address this problem splines are fitted to model the road in a way in which they capture the most information about the road centreline. Similar to the approach to coast line modelling in [33] this work adopts the B-splines fitting described in [34] for road centreline modelling. Here we improve the road modelling by adjusting the locations of the spline nodes to reflect the curvature of the road as follows. First a spline is fitted to road branch pixels to provide filtered coordinates of the branch.

The curvature of the obtained filtered branch is analysed ${ }^{1}$ by first fitting polygons to the points and then calculating the analytical curvature between consequent points of the polygons. Figure 6 (bottom) illustrates the process of curvature accumulation, where the location of the nodes on the branch are marked with red lines. The curvature threshold is set based on scale of the image, with lower threshold for images with lower scale (taken at low altitudes) and higher threshold for images taken from higher altitudes, that allows recording of all significant changes in road direction and discards the insignificant fluctuations due to the presence of occlusions.

Modelling road branches with splines is an effective method of converting the pixel information into scale independent form, since a spline describes the shape of the feature independent of the scale at which the feature is observed. Splines also minimise

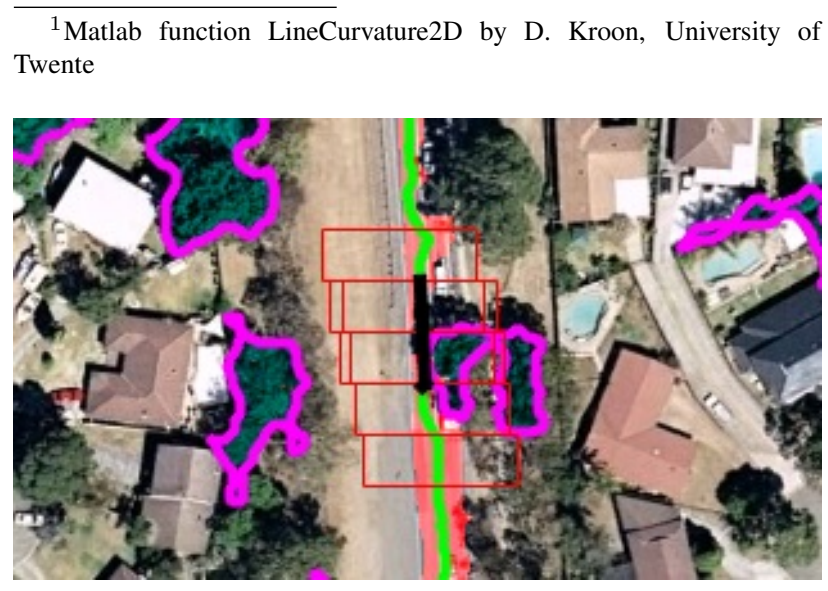

Figure 5: Image of extrapolation search (red rectangles) for selected road branches (green)

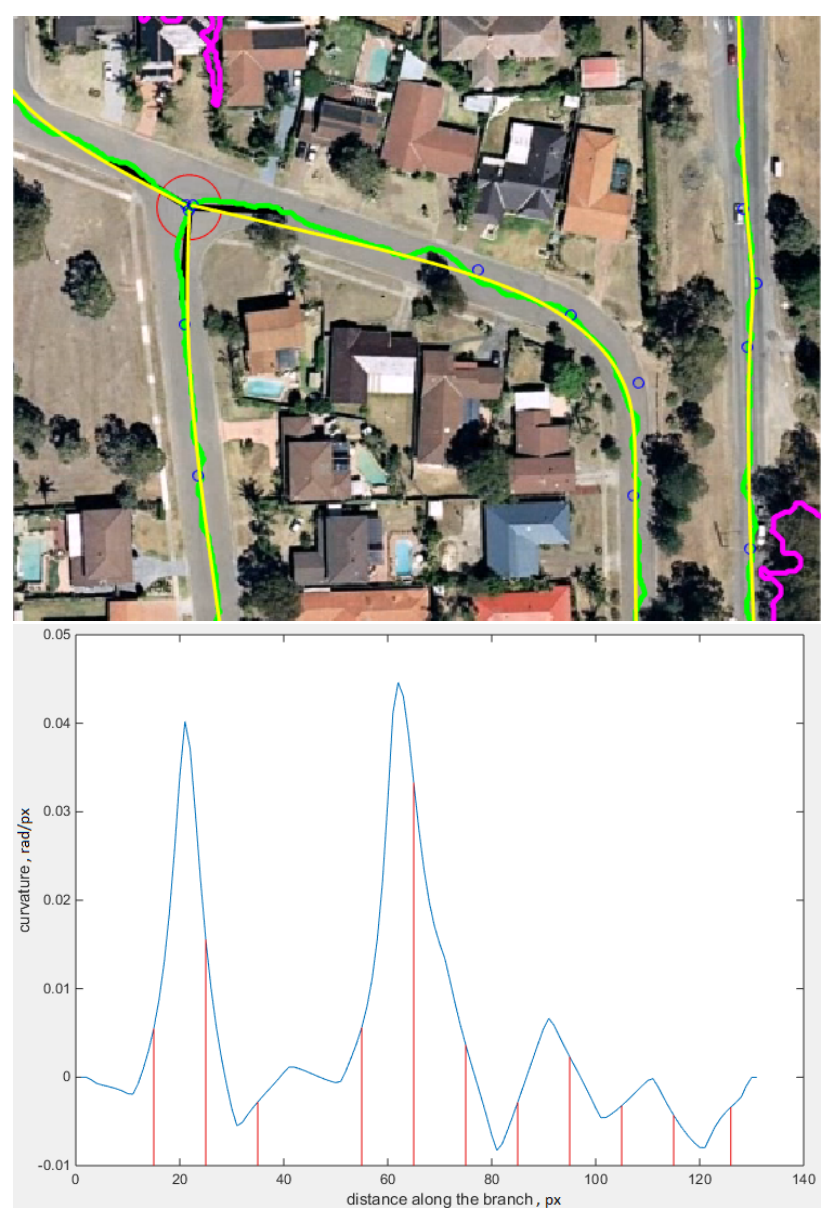

Figure 6: (top) Road branch points generated by thinning (green) are modelled with a spline (yellow); (bottom) curvature of the road branch with locations of the nodes shown in red

the amount of information with which the feature is encoded and therefore are preferable for feature matching and data association.

3.3.3 Post-processing of the junctions Road intersections are prioritised in data association because correct registration of a road intersection constrains the position of the observer in both directions, compared to straight sections of the road, which can only constrain the position in direction normal to its centreline. Hence, accurate detection and extraction of information about intersections present in the image is of primary importance. Distortion in the locations of road intersections due to imperfection in the skeletonisation operation is one of the common problems in road extraction. This problem has been recently approached by substituting the skeletonisation procedure with tensor voting [13, $16,26,35]$, which is superior to traditional methods in extracting the geometrical structures but much more computationally demanding and may therefore be unsuitable for real-time applications. In this paper the problem of distorted junctions is approached from a feature association perspective and a composite solution suitable for road-extraction is offered. A feature matching algorithm, operating on features described above, matches junctions by their location, number of branches and branch angular distribution, and branch absolute angles, allowing for some tolerance. The problem of junctions being offset and the branch angles being skewed becomes crucial as it can generate false positives or simply does not allow for data associations. Rather than relaxing the matching constraints to improve the representation of the junctions several post-processing steps described below are applied. 


\section{T-junctions}

The skeletonisation operation often causes the offset of the Tjunction centroid in the direction of the connecting branch (Fig.7). Since the junction database or map will have T-junctions with branches intersecting at angles close to $180^{\circ}$ or $90^{\circ}$ and one part being straight, the intersections detected from the splines should be adjusted. The new centroid of a road junction is formed by finding an intersection of the branches based on nodes located around the junction.

\section{Revision of $T$ - and $X$ - junctions}

The spline-generating algorithm may cause the loss of road intersections due to a tendency to directly join the road branches. To avoid this situation and ensure repeatability of the feature detection method, the cases where a spline joins the branches of the junction are revisited by a post-processing routine. The possible location and distribution of branches of such junctions is determined based on the curvature and mutual location of neighbouring splines (Fig.7). A typical application of the road network detection algorithm is shown in Fig. 8.

\subsection{Road feature registration}

After a feature has been detected in the image, information capturing its uniqueness was extracted and stored in a database. Since parts of a road network detected in the image need to be analysed and compared with the database individually, the road segments are stored separately from road intersections. Information about the connectivity of road components is stored in a database index. This section overviews encoding of road centrelines and intersection detected previously for database construction. Choice of the coordinate system to store the road database was made taking into account the environment the system operated in. The geodetic frame was chosen, which means that the location of spline nodes and intersection centroids were converted from camera frame into ECEF reference frame.

3.4.1 Road centreline feature Let $R$ represent a database entry corresponding to the road centreline

$$
R=[X, Y, Z]_{s}^{g}, w_{r}, n_{i}, i_{i} .
$$

The parameters associated with it are 1) the location of $s$ spline nodes $[X, Y, Z]_{s}^{g}$ representing road centreline, 2 ) the average width of the road region $w_{r}$ calculated perpendicular to the road centreline, 3 ) the number of intersections $n_{i}$ road segment connects to,
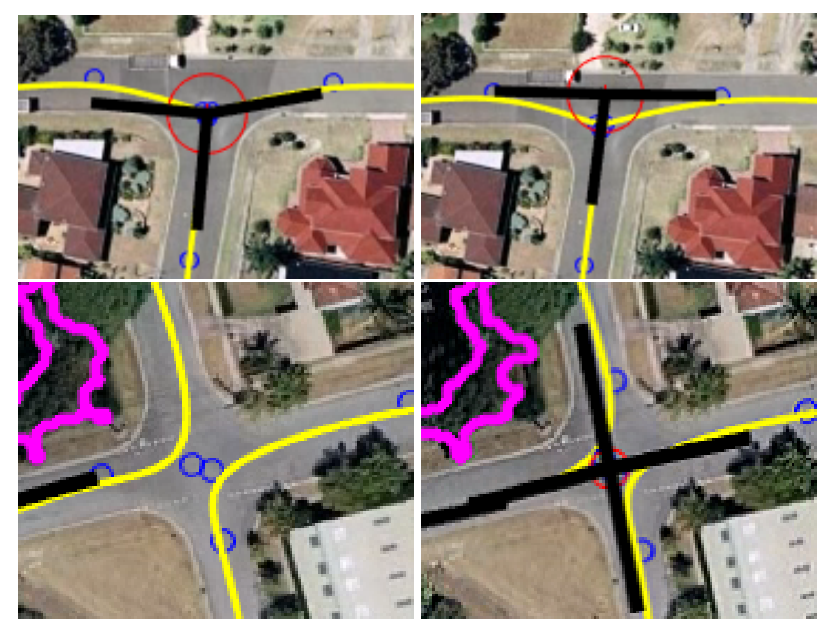

Figure 7: Typical cases of T- and X-junction revision: junction centres are shown as red circles and branches are shown as black lines.
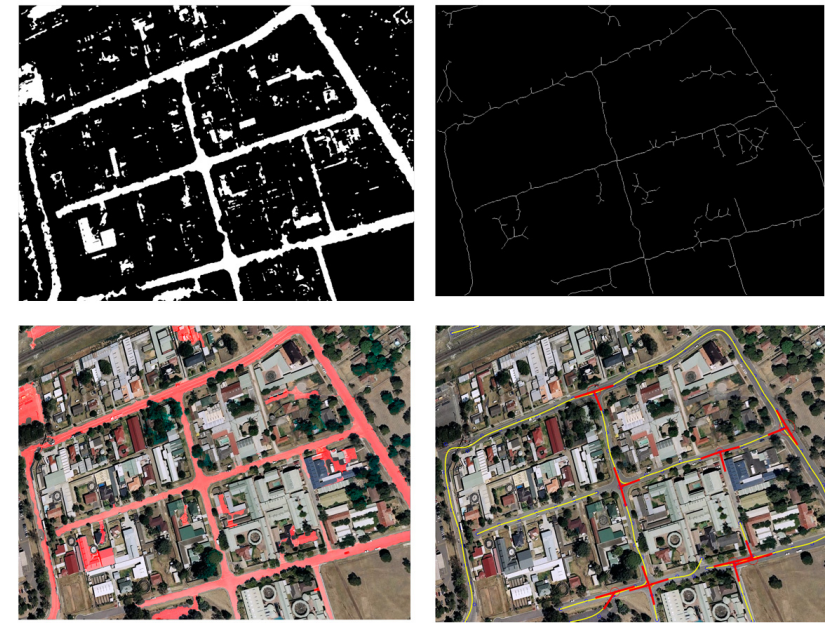

Figure 8: Road extraction example stages: (a) raw road segmentation, (b) road components superimposed on the original image, (c) road skeleton, (d) road network with road centrelines shown in yellow and intersections in red.

4) the indices of intersections associated with the road segment $i_{i}$.

3.4.2 Road intersection feature The road intersection feature modelling approach used here was adopted from Dumble [36]. Intersection descriptor $I$, that permits performing intersection matching regardless of the position and orientation of the feature in the camera frame, looks as follows.

$$
I=[X, Y, Z]^{g}, n_{b}, \psi_{b N}, \psi_{b},
$$

where $[X, Y, Z]^{g}$ is the location of the intersection centroid in the geodetic frame $F_{g}, n_{b}$ is the number of road branches, $\psi_{b N}$ angles of the road branches forming the intersection relative to North (Fig. 9) and $\psi_{b}$ - the angular difference between the successive road branches. The width of the branches can also be added to the descriptor to improve uniqueness of the feature.

\section{FEATURE LOCALISATION AND ASSOCIATION}

\subsection{Road network feature matching}

Feature matching is the part of the visual navigation algorithm responsible for associating the features detected in the camera frame with those in a database. Improvement of both the uniqueness of features and the construction of several association levels ensures fault detection prior to feeding the feature into the navigational update. To optimise the computational load of the association operation on the system, the matching tasks are prioritised and assigned to different threads, each associated with a specific
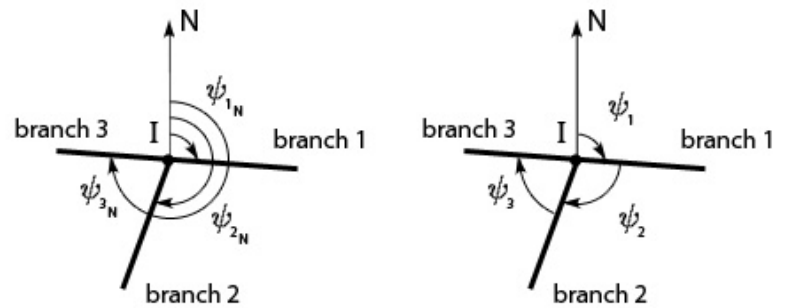

Figure 9: Road Intersection branch labelling and angle determination 

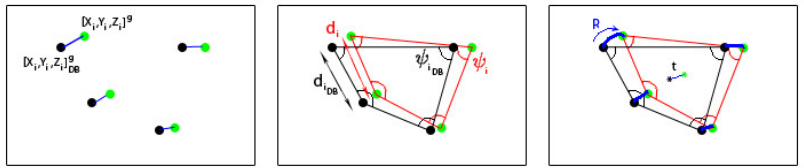

Figure 10: Pattern matching and transformation calculation for intersections detected in the camera (green dots) and database matches (black)

combination of features present in the image. Choice of data association thread depends on both type and number of features present in the camera frame. The correspondence between features present in the camera frame and the initialised data matching threads is shown in Table 1.

\begin{tabular}{|c|c|c|}
\hline & Feature combination & Matching thread \\
\hline 1 & roads and 1+ intersections & intersection pattern matching \\
\hline 2 & roads and 1 intersection & intersections and splines \\
\hline 3 & roads only & splines \\
\hline
\end{tabular}

Table 1: Correspondence between features present in the frame and initiated association thread

The choice of features for data association is hierarchical and can be explained by differences in priority of the operations. As mentioned before, intersection matching has priority compared to spline matching because it is less computationally expensive and provides absolute information constraining the inertial drift from the IMU. Hence, if the intersection association thread initiated in the first case (Table 1.), successfully registers the pattern of intersections in the database, there is no need for additional spline matching. In the third case, however, when intersection association is not possible, localisation information is derived from spline matching only. Depending on the shape of the spline, it can provide precision in one or two directions. Therefore, splines with sections of high curvature can be seen as unique features are assigned higher priority in the matching sequence over virtually straight splines. Future realisation of the data association algorithm will also consider water bodies and shapes formed by greenery detected in the frame in pattern analysis. Data association threads are described in the next section.

\subsection{Road intersection matching}

4.2.1 Intersection feature matching Each of the intersections detected in the camera frame is matched to the feature database based on the ECEF coordinates $\left[X_{i}, Y_{i}, Z_{i}\right]^{g}$, number of branches $n_{b}$, and angles between them. Pairs of intersections for which the least-square position error $\delta L_{i}$ and the difference of orientation of the branches $\psi_{i}$ are lower than the corresponding thresholds are considered as a potential match. The corresponding comparison measures are defined as follows.

$$
\begin{gathered}
n_{i}=n_{i_{D B}} ; \delta \psi_{i}=\Sigma_{i}^{n}\left(\psi_{i}-\psi_{i_{D B}}\right) ; \\
\delta \mathrm{Ł}_{i}=\sqrt{\left.\left.\Sigma\left(\left[X_{i}, Y_{i}, Z_{i}\right]\right)^{g}-\left(\left[X_{i}, Y_{i}, Z_{i}\right]_{D B}^{g}\right)\right)^{2}\right)}
\end{gathered}
$$

Depending on the number of intersections in the frame pattern matching is initiated, which compares the angles and the distance of the polygon constructed from camera features (green dots, Fig.10) to those of the polygon constructed using their database matches (shown with black dots).

4.2.2 Intersection pattern matching At this stage the angle $\psi_{i}$ formed by vertex $i$ and distance between the adjacent vertices $d_{i}$ is compared with the corresponding angle and distance in the polygon built based on the database information and the matches which produce errors $\delta \psi_{i}, \delta d_{i}$ higher than the threshold value are rejected. Errors $\delta \psi_{i}, \delta d_{i}$ are defined as

$$
\begin{gathered}
\delta \psi_{i}=\Sigma\left(\psi_{i}-\psi_{i_{D B}}\right) \\
\delta d_{i}=\Sigma\left(d_{i}-d_{D B}\right)
\end{gathered}
$$

The check of the angles and distances a pattern forms ensures that the detected features are located in the same plane and connection between them resembles the pattern stored in the database. After correspondence between the matched features being confirmed, the transformation between the corresponding vertices of the two polygons (Fig.10) is estimated through singular value decomposition to correct the camera pose. Since the offset remains consistent for all features in the frame, the transformation defined by rotation matrix $R$ and translation vector $t$ estimated via pattern matching can serve as an update for the Kalman filter. Precision of the aircraft estimate is generally sufficient to isolate possible matches within the database so, repetitive patterns and regular geometry is not considered to be a problem. If multiple possible matches cannot be discriminated, none of them will be used as innovations.

4.2.3 Road centreline matching The spline nodes accurately capture the location of the road centreline and information about the shape of the road component. It would be incorrect though to match the location of individual nodes of the splines present in the image to the ones in the database due to the non-deterministic nature of the procedure though which they are generated. However, spline matching can reliably use the characteristic shape of the road segment by analysing its curvature. Spline matching takes into account the peculiarity that spline features have due to aerial video as their sources: each subsequent piece of the information about the feature "enters" the frame at the top, and is added to the feature representation available from the previous frame.

Curvature-based spline matching uses algebraic curvature description of the spline to search for correspondences in the database. Once the correspondence is found, the part of the feature which enters the camera field of view in the subsequent frame, is added to the match correspondingly. The spline matching procedure, depending on the shape of the road, can constrain the drift of dead reckoning in one or both directions. This leads to priority ranging of detected splines. The sections capturing grater change in curvature of a spline will have higher priority in the matching sequence since they constrain the drift in both directions in a 2D plane compared to relatively straight sections of the splines which can only limit the drift of the vehicle in a direction perpendicular to the spline. Two subsequent video frames with spline sections limiting the position drift in both directions are shown as an example in Figure 11. It is worth noting that the repeatability of the spline extraction across the frames allows reliable operation of both SLAM and database matching threads.

\section{EXPERIMENTAL RESULTS}

The algorithm was implemented in Matlab, on a 3.6Ghz Intel i7 4 core processor. A relatively low resolution of the Google Imagery $(1024 x 768 \mathrm{px})$ was deliberately chosen to minimise the differences between the simulation and real imagery. For future implementation of the system, the cameras on the UAV will be chosen with respect to the requirements of the system. Possible blur and stabilisation issues occurring in the real sequence are planned to be addressed with additional processing modules of the algorithm. For the purpose of testing the algorithm, Google Earth projected imagery was taken with no consideration of terrain height variation. This has some effect on accumulation of 


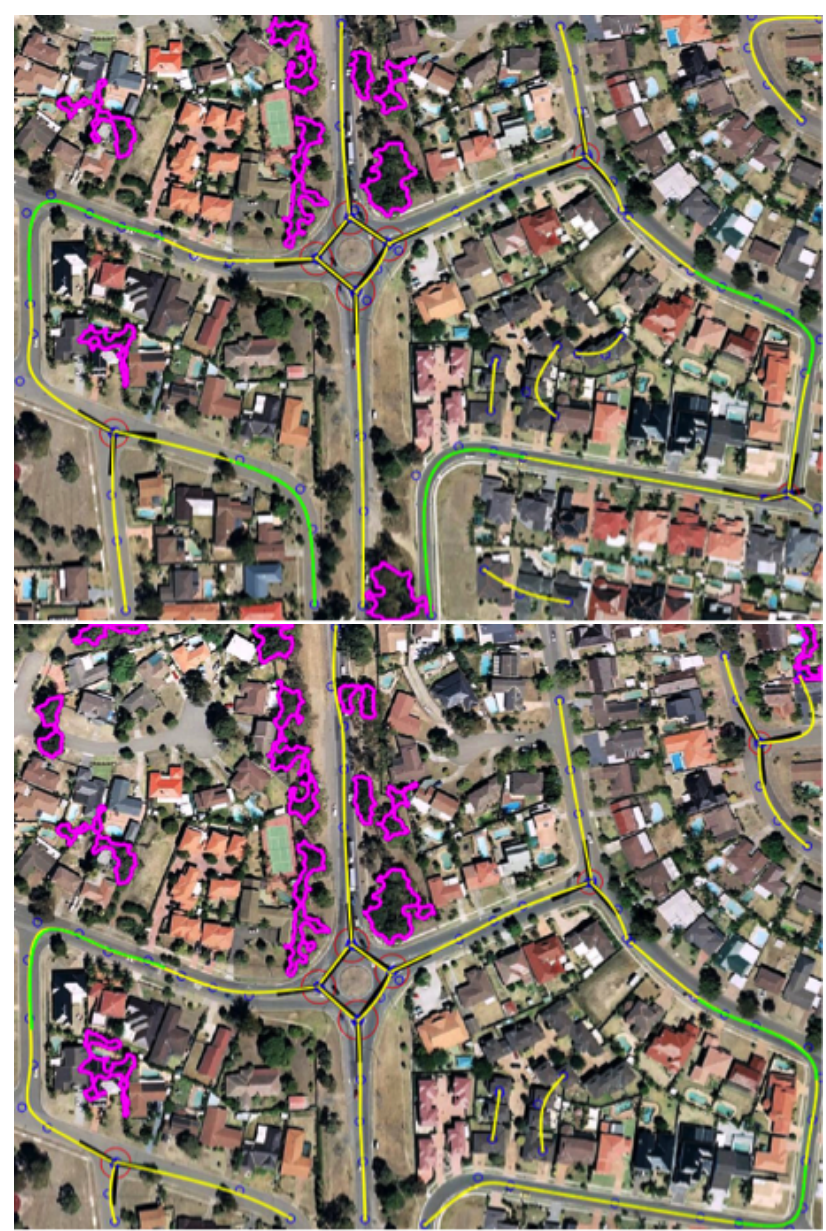

Figure 11: Splines detected in the frame (shown in yellow) compared to priority matching spline (green).

the error in altitude (see Fig. 14). For future processing of real UAV flight data, compensation of the range to the surface and terrain height using Digital Elevation Maps will be added to the

\begin{tabular}{|c|c|c|c|}
\hline Dataset & Features detected & Features matched & Ratio \\
\hline Dataset 2007 & 566 & 152 & $27 \%$ \\
\hline Dataset 2009 & 166 & 46 & $27 \%$ \\
\hline Dataset 2014 & 768 & 150 & $20 \%$ \\
\hline
\end{tabular}

Table 2: Comparison of number of features detected and fused in the navigation filter

\section{algorithm.}

A number of tests were conducted to evaluate the robustness of the algorithm. Three datasets based on Google Earth imagery taken in different years $(2007,2009$, and 2014) closely resemble video that would typically be taken from an on-board downward looking camera, including variations in camera field of view when the vehicle is performing a coordinated turn. The three datasets were picked to represent different season, lighting conditions as well as to capture structural changes of the urban environment (Fig. 12). All three videos were analysed by feature extraction and matching threads of the algorithm. A database of intersections used for feature matching was constructed separately by manually extracting the locations and angular orientations of the road intersections in the fly-over area using Google Earth software. Criteria for positive matches were chosen as angle $\delta \psi_{i}<2^{\circ}$, and distance $\delta Ł_{i}<\delta Ł_{t h r}$, where $\delta Ł_{t h r}=8[m]$, to ensure fusion of only true positives in the navigational Kalman filter (for description of the criteria see 4.2.1). As a planar accuracy measure, the distribution of distance and difference in angular orientations of matched junctions from Dataset 2007 is presented on Fig 13. The comparison of the number of features identified and matched per dataset is shown in Table 2.

The position drift accumulated during flight with updates provided by VNS is shown in North, East and down directions (Fig. 14). The number of intersections detected in the image and used for data fusion compared with the number of features in the database is shown in Fig. 15. The effect of varying lighting and seasonal conditions is reflected in the difference between the numbers of detected features in different videos compared. Although the number of features and regularity of updates is lower for Dataset 2009 compared to the other two datasets, the corresponding navigational update proves that the algorithm is able to constrain the inertial drift even with relatively infrequent updates.
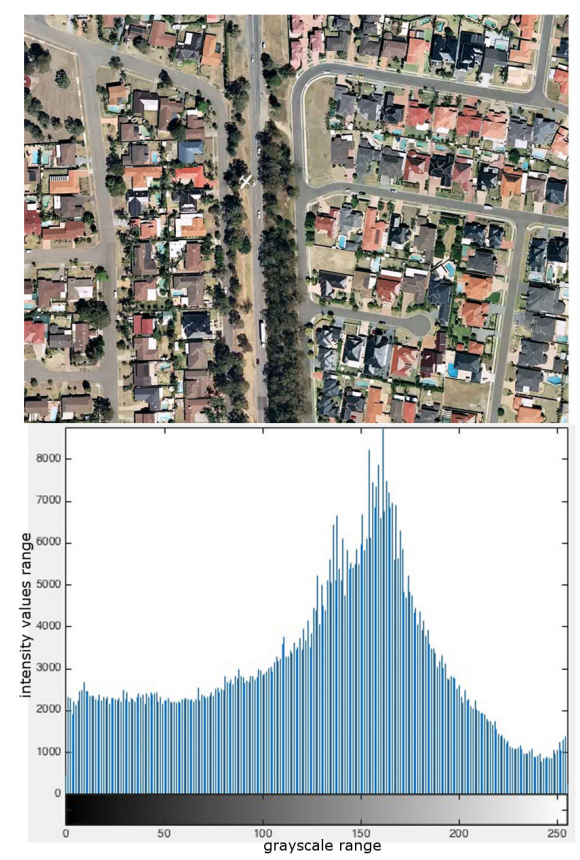
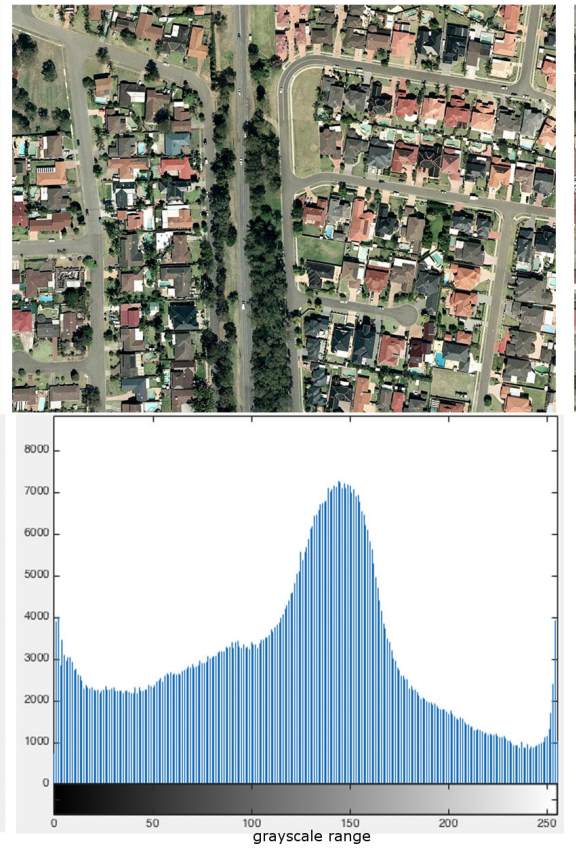

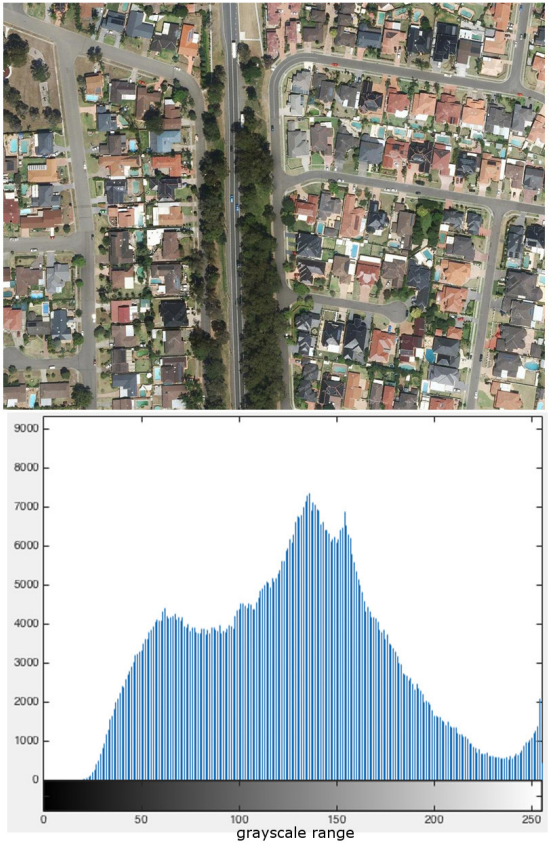

Figure 12: Combined histograms of the frame \#1 from Datasets 2007, 2009, 2014 correspondingly. 

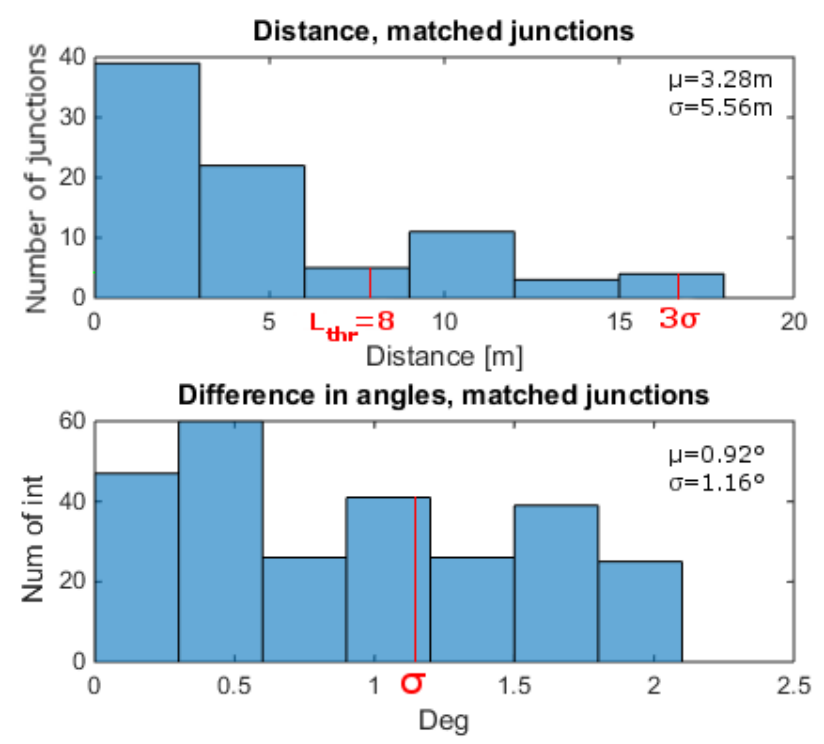

Figure 13: Accepted errors in distance and angular orientation of the matched intersections shown against the number of intersections.

The sections of the video between frames 90-100 and 154-180 correspond to flight over the area covered by the lake and forest respectively. No intersections or road centrelines are detected within these sections of the video that corresponds to the period of unconstrained position drift (Fig. 14). From frame 200, the airplane enters an urban area and as soon as the positive reliable match is found, the position error drops to a value close to zero. Other peculiarities connected to the dataset account for structural changes, such as the presence of the new built-up area in frames 149-153 of the 2014 dataset, which were not present at the time of the database construction.

The breakdown of execution time, showing the share of each of the functions in the overall processing time of a typical 1024x 768 px frame from an aerial sequence, is presented in Table 3.

\begin{tabular}{|c|c|c|}
\hline Algorithm module & Time, $[\mathrm{s}]$ & Ratio \\
\hline Extraction & 0.0820 & $7.4 \%$ \\
\hline Detection & 0.6791 & $61 \%$ \\
\hline Association & 0.3444 & $31 \%$ \\
\hline Other & 0.0067 & $0.6 \%$ \\
\hline Total & 1.1055 & $100 \%$ \\
\hline
\end{tabular}

Table 3: Breakdown of the algorithm execution time for a typical $1024 \times 768$ px video frame

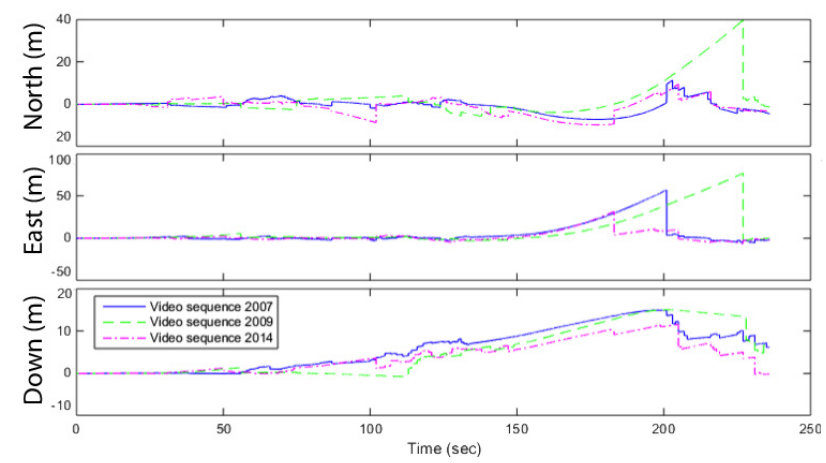

Figure 14: Position error between true vehicle position and the position calculated from IMU data integrated with VNS in North, East and down directions.

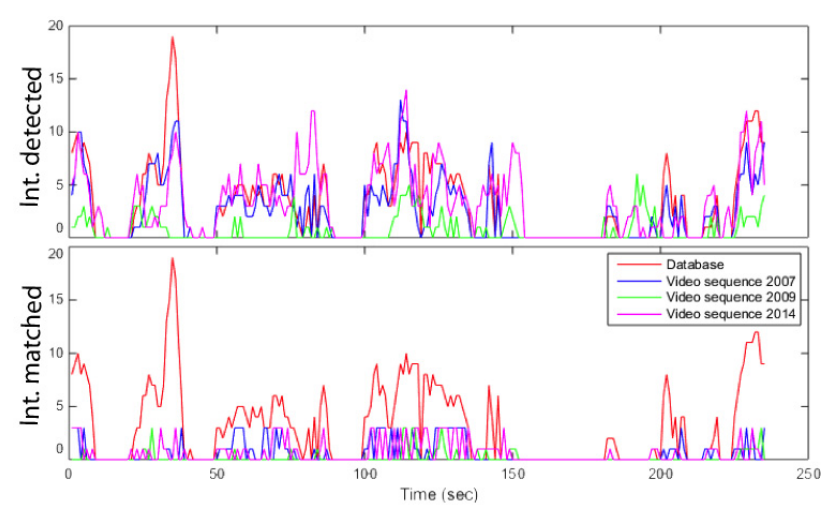

Figure 15: The number of features detected and matched with the database in the videos sequences generated using Google Earth imagery from 2007, 2009, and 2014.

\section{CONCLUSION}

Current work shows the effective application of the designed feature extraction and matching algorithm to the task of visual navigation. Feature extraction technique aimed to maximise the uniqueness of each detected feature and a frame as a whole. The feature description and registration techniques developed use minimal description vector to optimise the operation of the matching system performing a continuous database search, producing reliable periodic position update.

Testing of the algorithm performance in the presence of varying image conditions, such as changes in illumination and seasonal effect, has proved that an intensity-based classifier combined with frequency information can present a reliable robust solution for region extraction. The comparison has shown the effect of the change in intensity of the image on feature detection. The drop in the number of features detected in the most recent sequence (Dataset 2014) with least contrast resulted in less frequent navigational updates although with no significant loss of accuracy. The test also proved that the system operates reliably with only $20-30 \%$ of the features detected from those present in the image without drop in accuracy of the localisation solution. From the presented graphs it is evident that the localisation error drops significantly each time the features are detected and registered in the image. The database search also allows for prolonged periods with no features detected, by adapting the search region of the database according to the position uncertainty.

The contributions of this paper are the multi-pronged approach to feature detection and the design of the automatic road feature extraction and matching (ARFEM) algorithm. They will serve as a basis for the development of future feature-based navigation algorithms for visual navigation. Ongoing work on the algorithm includes the integration of Optical Flow [37] to provide a Kalman update of the vehicle speed in $x$ and $y$ directions based on the motion of the objects found in the camera frame. With direct update of the velocity estimates derived from Optical Flow, the drift rate of the inertial navigation system will change linearly rather than in a quadratic fashion typical of double integration of acceleration information from the inertial sensors. Further algorithm improvement will include development of multi-temporal operation modes for the feature extraction and matching modules as well as the use of contextual information to improve the reliability of feature extraction. Current test and evaluation of the algorithm using real flight test imagery in under way. The focus of this work is on the evaluation of the performance of the image processing components in presence of variations of natural lighting and changes in the urban environment from the Google datasets. 


\section{ACKNOWLEDGEMENTS}

The author wishes to thank Dr. Steve J. Dumble and David G. Williams whose research inspired and contributed to the current work.

\section{REFERENCES}

[1] A. Cesetti, E. Frontoni, A. Mancini, P. Zingaretti, and S. Longhi, "A vision-based guidance system for UAV navigation and safe landing using natural landmarks," in Selected papers from the 2nd International Symposium on UAVs, Reno, Nevada, USA June 8-10, 2009, 2010, pp. 233-257.

[2] A. Marburg, M. P. Hayes, and A. Bainbridge-Smith, "Pose Priors for Aerial Image Registration," in International Conference on Digital Image Computing: Techniques and Applications (DICTA), 2013, pp. 1-8.

[3] A. Cesetti, E. Frontoni, A. Mancini, P. Zingaretti, and S. Longhi, "A Vision-Based Guidance System for UAV Navigation and Safe Landing using Natural Landmarks," Journal of Intelligent and Robotic Systems, vol. 57, pp. 233-257, 2009.

[4] C. Forster, M. Pizzoli, and D. Scaramuzza, "SVO: Fast semidirect monocular visual odometry," in Conference on Robotics and Automation (ICRA), 2014 IEEE International , 2014, pp. 1522.

[5] J. B. Mena, "State of the art on automatic road extraction for GIS update: a novel classification," Pattern Recognition Letters, vol. 24, pp. 3037-3058, 2003.

[6] H. Mayer and S. Hinz, "A test of automatic road extraction approaches," 2006.

[7] A. Volkova and P. W. Gibbens, ”A Comparative Study of Road Extraction Techniques from Aerial Imagery: A Navigational Perspective," Asia-Pacific International Symposium on Aerospace Technology (APISAT), Cairns, Australia, 25 - 27 November 2015.

[8] F. Bonin-Font, A. Ortiz, and G. Oliver, "Visual Navigation for Mobile Robots: a Survey," 2008.

[9] M. Song and D. Civco, "Road Extraction Using SVM and Image Segmentation," 2004.

[10] J. Inglada, "Automatic recognition of man-made objects in high resolution optical remote sensing images by SVM classification of geometric image features," ISPRS Journal of Photogrammetry and Remote Sensing, vol. 62, pp. 236-248, 2007.

[11] X. Huang and L. Zhang, ”An SVM ensemble approach combining spectral, structural, and semantic features for the classification of high-resolution remotely sensed imagery," IEEE Transactions on Geoscience and Remote Sensing, vol. 51, pp. 257-272, 2013.

[12] X. Huang and Z. Zhang, "Comparison of Vector Stacking, Multi-SVMs Fuzzy Output, and Multi-SVMs Voting Methods for Multiscale VHR Urban Mapping," Geoscience and Remote Sensing Letters, IEEE, vol. 7, pp. 261-265, 2010.

[13] M. Zelang, W. Bin, S. Wenzhong, and W. Hao, "A Method for Accurate Road Centerline Extraction From a Classified Image," IEEE Journal of Selected Topics in Applied Earth Observations and Remote Sensing, vol. 7, pp. 4762-4771, 2014.

[14] C. Poullis, "Tensor-Cuts: A simultaneous multi-type feature extractor and classifier and its application to road extraction from satellite images," ISPRS Journal of Photogrammetry and Remote Sensing, vol. 95, pp. 93-108, 2014.

[15] Z. Sheng, J. Liu, S. Wen-zhong, and Z. Guang-xi, "Road Central Contour Extraction from High Resolution Satellite Image using Tensor Voting Framework," in 2006 International Conference on Machine Learning and Cybernetics, 2006, pp. 32483253.

[16] C. Cheng, F. Zhu, S. Xiang, and C. Pan, "Accurate Urban Road Centerline Extraction from VHR Imagery via Multiscale
Segmentation and Tensor Voting," IEEE Transactions on Geoscience and Remote Sensing, 2014.

[17] C. Wiedemann and H. Ebner, "Automatic completion and evaluation of road networks," International Archives of Photogrammetry and Remote Sensing, vol. 33, pp. 979-986, 2000.

[18] S. S. Shen, W. Sun, D. W. Messinger, and P. E. Lewis, "An Automated Approach for Constructing Road Network Graph from Multispectral Images," vol. 8390, pp. 83901W-83901W-13, 2012.

[19] A. W. Elliott, "Vision Based Landmark Detection For Uav Navigation," MRes Thesis, 2012.

[20] C. Steger, C. Glock, W. Eckstein, H. Mayer, and B. Radig, "Model-based road extraction from images," 1995.

[21] A. Barsi, C. Heipke, and F. Willrich, "Junction Extraction by Artificial Neural Network System - JEANS," 2002.

[22] G. Duo-Yu, Z. Cheng-Fei, J. Guo, S.-X. Li, and C. HongXing, "Vision-aided UAV navigation using GIS data," in Vehicular Electronics and Safety (ICVES), 2010 IEEE International Conference on, 2010, pp. 78-82.

[23] O. Besbes and A. Benazza-Benyahia, "Road Network Extraction By A Higher-Order CRF Model Built On Centerline Cliques." [24] M. Nieuwenhuisen, D. Droeschel, M. Beul, and S. Behnke, "Autonomous MAV Navigation in Complex GNSS-denied 3D Environments," 2015.

[25] M. Blsch, S. Omari, M. Hutter, and R. Siegwart, "Robust Visual Inertial Odometry Using a Direct EKF-Based Approach." [26] Z. Miao, W. Shi, H. Zhang, and X. Wang, "Road centerline extraction from high-resolution imagery based on shape features and multivariate adaptive regression splines," IEEE, 2012.

[27] G. Conte and P. Doherty, "An Integrated UAV Navigation System Based on Aerial Image Matching," p. 10, 2008.

[28] C.-F. Zhu, S.-X. Li, H.-X. Chang, and J.-X. Zhang, "Matching road networks extracted from aerial images to GIS data," in Asia-Pacific Conference on Information Processing, APCIP 2009, pp. 63-66.

[29] W. Liang and H. Yunan, "Vision-aided navigation for aircrafts based on road junction detection," in IEEE International Conference on Intelligent Computing and Intelligent Systems ICIS, 2009.. , 2009, pp. 164-169.

[30] J. Jung, J. Yun, C.-K. Ryoo, and K. Choi, "Vision based navigation using road-intersection image," in 11th International Conference on Control, Automation and Systems (ICCAS), 2011, pp. 964-968.

[31] A. Dawadee, J. Chahi, and D. Nandagopal, ”An Algorithm for Autonomous Aerial Navigation Using Landmarks," Journal of Aerospace Engineering, vol. 0, p. 04015072, 2015.

[32] R. C. Gonzalez, R. E. Woods, and S. L. Eddins, Digital Image processing using MATLAB. United States: Gatesmark Publishing, 2009.

[33] D. G. Williams and P. W. Gibbens, "Google Earth Imagery Assisted B-Spline SLAM for Monocular Computer Vision Airborne Navigation."

[34] L. Pedraza, G. Dissanayake, J. V. Mir, D. Rodriguez-Losada, and F. Matia, "BS-SLAM: Shaping the World," in Robotics: Science and Systems, 2007.

[35] C. Poullis and S. You, "Delineation and geometric modeling of road networks," in ISPRS Journal of Photogrammetry and Remote Sensing, vol. 65, pp. 165-181, 2010.

[36] S. J. Dumble and P. W. Gibbens, "Airborne Vision-Aided Navigation Using Road Intersection Features," JINT, 2014.

[37] B. K. Horn and B. G. Schunck, "Determining optical flow," in 1981 Technical symposium east, 1981, pp. 319-331. 